\title{
Axial irradiance for spherically aberrated holographic optical elements
}

\author{
A. Beléndez \\ Departamento de Ingeniería de Sistemas y Comunicaciones, Universidad de Alicante, Apartado 99, E-03080, Alicante, Spain
}

\author{
L. Carretero and A. Fimia
}

Laboratorio de Optica, Departamento Interuniversitario de Optica, Universidad de Alicante, Apartado 99, E-03080, Alicante, Spain

Received February 16, 1994

The effect of third-order balanced spherical aberration on the axial irradiance of holographic lenses is considered. It is shown that the number of principal maxima of axial irradiance increases for large aberrations, and the position of these points can be different from the position of the minimum aberration variance point.

The properties and characteristics of the axial irradiance of optical systems have been the subject of several papers published over the past few years. ${ }^{1-9}$ When aberrations are present the principal maximum of the axial irradiance lies not at the Gaussian image point but at an axial point closer to it (for large Fresnel numbers). However, the axial irradiance distribution has not been sufficiently studied for optical systems with large aberrations, such as holographic optical elements. In this Letter we obtain the axial irradiance for holographic lenses (HL's) with a circular pupil (and analyze certain properties of this irradiance) from a knowledge of its third-order spherical aberration. We consider HL's in the presence not only of small but also of large spherical aberrations.

For simplicity, we consider a spherically aberrated in-line HL with a uniformly illuminated circular pupil with a diameter $D$. Its axial irradiance at a distance $z$ from the exit pupil plane of the holographic lens, $I(z)$, i.e., the irradiance distribution along the line joining the center of the hologram and the Gaussian image point ( $z$ axis for an in-line HL), may be written $\mathrm{as}^{5}$

$$
I(z)=4\left(\frac{z_{g}}{z}\right)^{2}|U(z)|^{2}
$$

where

$$
U(z)=\int_{0}^{1} \exp [i 2 \pi \Phi(\rho, z)] \rho \mathrm{d} \rho
$$

In Eqs. (1) and (2), $z_{g}$ determines the position of the Gaussian image point, $\rho$ is in units of $D / 2$, and $\Phi(\rho, z)$ is the phase aberration. The normalization factor $4\left(z_{g} / Z\right)^{2}$ in Eq. (1) ensures that we have unit irradiance at the Gaussian image point in the absence of aberrations. For third-order spherical and defocus aberrations, $\Phi(\rho, z)=W_{20} \rho^{2}+W_{40} \rho^{4}$, where $W_{20}=F D^{2} / 8 \lambda_{c}$ denotes the defocus coefficient and is a function of $z$, and $W_{40}=-S D^{4} / 128 \lambda_{c}$ denotes the coefficient for third-order spherical aberration. $W_{40}$ and $W_{20}$ are in units of the wavelength. $S$ and $F$ are given by ${ }^{10}$

$$
\begin{aligned}
& S=\frac{1}{z_{c}^{3}}-\frac{1}{z_{g}^{3}}+\mu\left(\frac{1}{z_{o}^{3}}-\frac{1}{z_{r}^{3}}\right), \\
& F=\frac{1}{z_{g}}-\frac{1}{z},
\end{aligned}
$$

where $\mu$ is the quotient between the reconstruction, $\lambda_{c}$, and the recording, $\lambda_{r}$, wavelengths and $z_{r}, z_{o}, z_{c}$, and $z_{g}$ are the coordinates of the reference, object, reconstruction, and Gaussian image point sources, respectively. With the following change in the variable:

$$
u=\left\{\begin{array}{cc}
2 \sqrt{W_{40}} \rho^{2}+\frac{W_{20}}{\sqrt{W_{40}}} & \text { if } W_{40}>0 \\
2 \sqrt{-W_{40}} \rho^{2}-\frac{W_{20}}{\sqrt{-W_{40}}} & \text { if } W_{40}<0
\end{array},\right.
$$

Eq. (2) can be rewritten as

$$
\begin{aligned}
U(z)= & \frac{1}{4 \sqrt{\left|W_{40}\right|}} \exp \left(-i \pi W_{20}^{2} / 2 W_{40}\right) \\
& \times \int_{u_{1}}^{u_{2}} \exp \left( \pm i \pi u^{2} / 2\right) \mathrm{d} u
\end{aligned}
$$

where

$$
u_{1}= \pm \frac{W_{20}}{\sqrt{\left|W_{40}\right|}}, \quad u_{2}=2 \sqrt{\left|W_{40}\right|} \pm \frac{W_{20}}{\sqrt{\left|W_{40}\right|}}
$$

and + and - refer to the situations for which $W_{40}>0(S<0)$ and $W_{40}<0(S>0)$, respectively. The axial irradiance $I(z)$ can be obtained analytically from Eqs. (1), (6), and (7), and when the integration is carried out the result obtained is

$$
\begin{aligned}
I(z)= & \frac{1}{4\left|W_{40}\right|}\left(\frac{z_{g}}{z}\right)^{2}\left\{\left[C\left(u_{2}\right)-C\left(u_{1}\right)\right]^{2}\right. \\
& \left.+\left[S\left(u_{2}\right)-S\left(u_{1}\right)\right]^{2}\right\},
\end{aligned}
$$

where $C(\cdot)$ and $S(\cdot)$ are the Fresnel integrals. ${ }^{11}$ 
Now we determine the amount of defocus $W_{20}$ so that the variance of the phase aberration $\Phi(\rho, z)$ across the exit pupil of the HL is minimized, and we find that the optimum value is $W_{20}=-W_{40}$, or $F_{p}=S D^{4} / 16$, where $F_{p}$ is the coefficient of defocus evaluated at $z_{p}$ on the optical axis for which the variance is minimized, i.e., $F_{p}=\left(1 / z_{g}\right)-\left(1 / z_{p}\right)$. It is easy to obtain

$$
z_{p}=\frac{D^{2} z_{g}}{D^{2}+8 \lambda_{c} W_{40} z_{g}} .
$$

For a small spherical aberration, in the best focal plane ${ }^{5,12} W_{20}=-W_{40}$. If we make the following change in the variable $z^{\prime}=z-z_{p}$, we recognize that $z^{\prime}$ represents the algebraic distance from an arbitrary point $z$ to the point $z_{p}$ for which the variance of the phase aberration across the pupil is minimized. For HL's, the Fresnel number $N=D^{2} / 4 \lambda_{c} z_{g}$ is large, and therefore $z$ and $z_{p}$ must be nearly equal to $z_{g}$ for adequate image quality. If $D$ varies between 4 and $9 \mathrm{~cm}, \lambda_{c}=633 \mathrm{~nm}$, and $z_{g}=40 \mathrm{~cm}$, we have HL's with $f$-numbers between 10 and 4.44 , and $N$ takes values between 1600 and 8000 . For optical systems with large Fresnel numbers $(N \gg 10)$ it must be true that $z^{\prime} \ll z_{p}, z_{g}$, and, using $z^{\prime}=z-z_{p}$, we may write Eq. (4) in the approximate form

$$
F \approx F_{p}+\frac{z^{\prime}}{z_{p}^{2}}=-\frac{8 \lambda_{c} W_{40}}{D^{2}}+\frac{z^{\prime}}{z_{p}^{2}}
$$

Also, taking into account that $z_{g} / z \sim 1$, we replace $z$ with $z_{g}$ in the inverse square dependence on $z$ in Eq. (8), and we obtain the irradiance distribution in the form

$$
\tilde{I}\left(z^{\prime}\right)=\frac{1}{4\left|W_{40}\right|}\left\{\left[C\left(\tilde{u}_{2}\right)-C\left(\tilde{u}_{1}\right)\right]^{2}+\left[S\left(\tilde{u}_{2}\right)-S\left(\tilde{u}_{1}\right)\right]^{2}\right\},
$$

where $\tilde{I}\left(z^{\prime}\right)=I(z)$ and

$$
\begin{aligned}
& \tilde{u}_{1}=-\sqrt{\left|W_{40}\right|} \pm \frac{\left(D / z_{p}\right)^{2}}{8 \lambda_{c} \sqrt{\left|W_{40}\right|}} z^{\prime} \\
& \tilde{u}_{2}=\sqrt{\left|W_{40}\right|} \pm \frac{\left(D / z_{p}\right)^{2}}{8 \lambda_{c} \sqrt{\left|W_{40}\right|}} z^{\prime}
\end{aligned}
$$

In Eqs. (12) the superscript corresponds to $S<0$, the subscript corresponds to $S>0$, and $\tilde{u}_{j}\left(z^{\prime}\right)=u_{j}(z), j=$ 1,2. It is easy to see that $\tilde{u}_{1}\left(-z^{\prime}\right)=-\tilde{u}_{2}\left(z^{\prime}\right)$, and then $\tilde{u}_{j}\left(z^{\prime}\right)=u_{j}(z), j=1,2$. It is easy to see that $\tilde{u}_{1}\left(-z^{\prime}\right)=-\tilde{u}_{2}\left(z^{\prime}\right)$, and then $\tilde{I}\left(z^{\prime}\right)=\tilde{I}\left(-z^{\prime}\right)$, i.e., the axial irradiance is symmetrical around $z_{p}\left(z^{\prime}\right)=0$, which corresponds to $W_{20}=-W_{40}$, and this point lies midway between the marginal and the Gaussian points obtained with ray tracing. For $z^{\prime}=0$, the axial irradiance is given by

$$
\tilde{I}(0)=\frac{1}{\left|W_{40}\right|}\left\{C^{2}\left(\sqrt{\left|W_{40}\right|}\right)+S^{2}\left(\sqrt{\left|W_{40}\right|}\right)\right\} .
$$

For example, if $W_{40}=1$, we obtain $\tilde{I}(0)=80 \%$.

When the expressions for the Fresnel integrals are considered and the cosine and the sine are expanded under the integral signs into power series and integrated term by term ${ }^{11}$ it is easy to see that, when $W_{40} \rightarrow 0, \tilde{I}(0)=1$, and it is clear that in the absence of spherical aberration the axial irradiance is one at the Gaussian image point. When the asymptotic approximations for the Fresnel integrals ${ }^{11}$ (which provide a good approximation to the integrals when the argument is large) are used in the following manner:

$$
\begin{aligned}
& C(u) \sim \frac{1}{2}+\left(\frac{1}{\pi u}\right) \sin \left(\frac{\pi u^{2}}{2}\right), \\
& S(u) \sim \frac{1}{2}-\left(\frac{1}{\pi u}\right) \cos \left(\frac{\pi u^{2}}{2}\right),
\end{aligned}
$$

Eq. (13) takes the approximate form

$$
\begin{aligned}
\tilde{I}(0) \approx & \frac{1}{\left|W_{40}\right|}\left\{\frac{1}{2}+\frac{1}{\pi^{2}\left|W_{40}\right|}\right. \\
& \left.+\frac{1}{\pi \sqrt{\left|W_{40}\right|}}\left[\sin \left(\frac{\pi}{2}\left|W_{40}\right|\right)-\cos \left(\frac{\pi}{2}\left|W_{40}\right|\right)\right]\right\} .
\end{aligned}
$$

This is an oscillatory function with a decreasing amplitude, and it is applicable for $\left|W_{40}\right| \geq 1.5$ with a relative error $\leq 5 \%$ with respect to the exact result obtained from Eq. (13).

We now apply the above equations to obtain some numerical results on the axial irradiance for in-line HL's in the presence of third-order spherical aberration. We analyze HL's recorded with a reference-divergent spherical wave and with an object-convergent spherical wave whose curvature centers are placed at $z_{r}=-120 \mathrm{~cm}$ and $z_{0}=60 \mathrm{~cm}$, respectively, and with a recording wavelength $\lambda_{r}=$ $633 \mathrm{~nm}$. The two source points are situated on the $Z$ axis normal to the HL plane. HL's are reconstructed with a collimated beam $\left(z_{c}=\infty\right)$ with a wavelength $\lambda_{c}=\lambda_{r}(\mu=1)$. For this readout geometry the Gaussian image point is situated at $z_{g}=40 \mathrm{~cm}$, and the image wave is convergent. This geometry ensures that the Gaussian image point (being a secondary image of the object point in a holographic sens $\mathrm{e}^{13}$ ) that is treated as an image of the reconstruction point is affected only by spherical aberration. Using Eq. (3), we obtain $S=-1.04167 \times 10^{-5} \mathrm{~cm}^{-3}$. Six different lens diameters are considered: $D=$ $4,5,6,7,8$, and $9 \mathrm{~cm}$, and for these diameters $W_{40}$ takes the values of $0.33,0.80,1.67,3.09,5.27$, and 8.44 (in units of the wavelength), respectively. From Eq. (9) we calculate the corresponding values for $z_{p}$ for each lens diameter $D$, and we obtain $z_{p}=39.9833$, $39.9740,39.9625,39.9490,39.9334$, and $39.9158 \mathrm{~cm}$ for $D=4,5,6,7,8$, and $9 \mathrm{~cm}$, respectively.

Figure 1 shows the axial irradiances as functions of $z^{\prime}$ for each lens diameter considered, calculated with Eqs. (12) and (13). As we can see from this figure, for small spherical aberrations (small values of $D$ ) we have only one point at which the adial irradiance is maximum, and this is the point $z^{\prime}=0$ with respect to which the variance of the phase aberration across the pupil is minimized. For these situations there is only one diffraction focus. ${ }^{7}$ However, when $D$ (and therefore spherical aberration as well) 

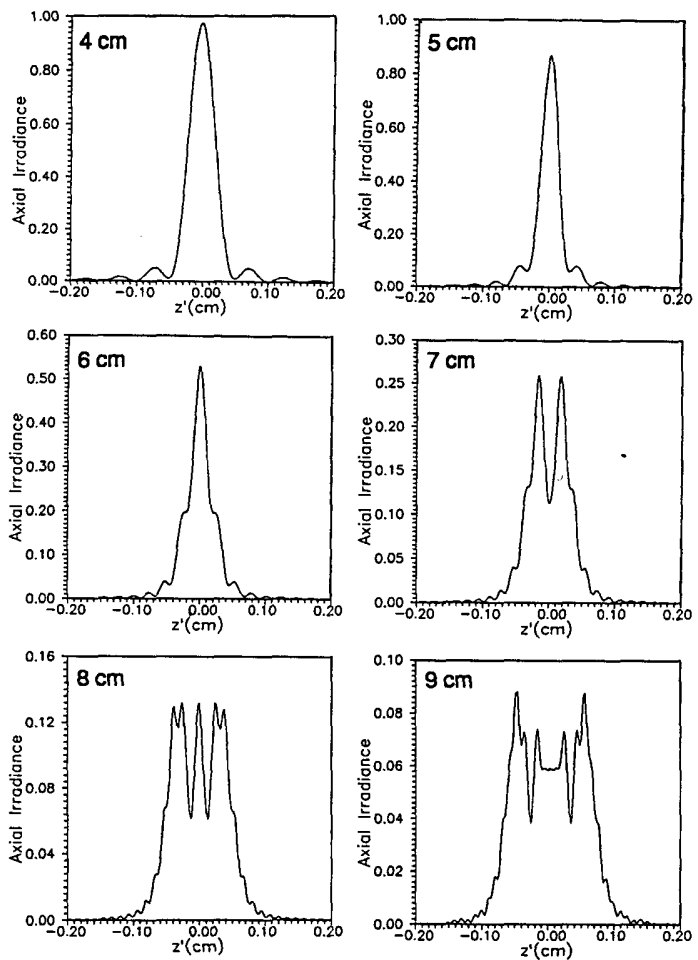

Fig. 1. Axial irradiance for different lens diameters.
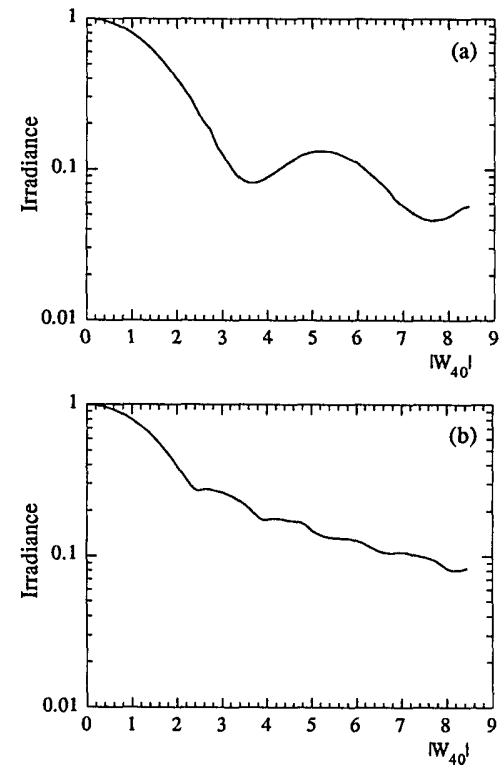

Fig. 2. (a) Irradiance for the point with respect to which the aberration variance is minimum, and (b) maximum irradiance obtained, both as functions of the modulus of the coefficient of spherical aberration $\left|W_{40}\right|$ (in units of the wavelength).

is increased, there are different points on the optical axis with maximum axial irradiance (there is more than one diffraction focus), and the number of these points increases when aberration increases too.
Also, at $z_{p}\left(z^{\prime}=0\right)$ the axial irradiance can take both maximum and minimum values, i.e., for large values of spherical aberration it is possible that $z_{p}$ may not be a diffraction focus, and then the best image plane is not placed in the $z=z_{p}$ plane. ${ }^{14}$ Figure 2 shows the irradiance for $z_{p}$ and for the maximum axial irradiance, both as functions of $\left|W_{40}\right|$. From Figs. 1 and 2 we can conclude that only for small spherical aberrations (up to a value of $W_{40}=\sim 2.3$ ) the irradiance is maximum at $z_{p}$ with respect to which the aberration variance is minimum. For large spherical aberrations, $z_{p}$ sometimes has a maximum irradiance value; however, this fact does not imply that $z_{p}$ determines the best image plane. ${ }^{15}$

Summarizing, in this Letter we describe the influence of third-order spherical aberrations on the axial irradiance of in-line HL's in terms of an analytic formula. We apply this study to recognize that an $\mathrm{HL}$ can have more than one diffraction focus for large values of spherical aberration. Also it is possible that the point with respect to which the aberration variance is minimum does not coincide with a point with maximum axial irradiance and only for a small spherical aberration $\left(W_{40} \leq 2.3\right)$ is the maximum of axial irradiance, at the point associated with the minimum aberration variance. Finally, the study presented in this Letter can also be extended to refractive lenses.

We would like to thank the reviewers of this letter for their useful suggestions, which significantly improved the original manuscript. We gratefully acknowledge the financial support of the Direcció General d'Ensenyaments Universitaris i Investigació (grant GV-1165/93), Generalitat Valenciana, Spain.

\section{References}

1. E. Collet and E. Wolf, Opt. Lett. 5, 264 (1980).

2. Y. Li and E. Wolf, Opt. Commun. 42, 151 (1982).

3. Y. Li, J. Opt. Soc. Am. 72, 770 (1992).

4. V. N. Mahajan, Appl. Opt. 22, 3042 (1983).

5. J. Ojeda-Castañeda, P. Andrés, and A. Díaz, Opt. Lett. 11, 487 (1986).

6. R. Torroba and M. Garavaglia, Appl. Opt. 26, 2860 (1987)

7. J. Ojeda-Castañeda, P. Andrés, and A. Díaz, J. Opt. Soc. Am. A 8, 1233 (1988).

8. J. Ojeda-Castañeda, E. Tepichín, and A. Pons, Appl. Opt. 27, 5140 (1988).

9. J. Ojeda-Castañeda, P. Andrés, and M. MartínezCorral, Appl. Opt. 31, 4682 (1992).

10. E. B. Champagne, J. Opt. Soc. Am. 57, 51 (1967).

11. M. Born and E. Wolf, Principles of Optics, 6th ed. (Pergamon, Oxford, 1980), pp. 430-431.

12. V. N. Mahajan, J. Opt. Soc. Am. 73, 860 (1983).

13. J. Nowak and M. Zajac, Optik 70, 143 (1985).

14. V. N. Mahajan, J. Opt. Soc. Am. 72, 1258 (1982).

15. L. Carretero, A. Fimia, and A. Beléndez, Opt. Lett. 19, 1355 (1994). 